\title{
An Outline of a Theory of Three-way Decisions
}

\author{
Yiyu Yao \\ Department of Computer Science, University of Regina, \\ Regina, Saskatchewan, Canada S4S 0A2 \\ E-mail: yyao@cs.uregina.ca
}

\begin{abstract}
A theory of three-way decisions is constructed based on the notions of acceptance, rejection and noncommitment. It is an extension of the commonly used binary-decision model with an added third option. Three-way decisions play a key role in everyday decision-making and have been widely used in many fields and disciplines. An outline of a theory of three-way decisions is presented by examining its basic ingredients, interpretations, and relationships to other theories.
\end{abstract}

\section{Introduction}

The concept of three-way decisions was recently proposed and used to interpret rough set three regions $[52,54,55]$. More specifically, the positive, negative and boundary regions are viewed, respectively, as the regions of acceptance, rejection, and noncommitment in a ternary classification. The positive and negative regions can be used to induce rules of acceptance and rejection; whenever it is impossible to make an acceptance or a rejection decision, the third noncommitement decision is made [54]. It can be shown that, under certain conditions, probabilistic three-way decisions are superior to both Palwak three-way decisions and two-way (i.e., binary) decisions [55]. Many recent studies further investigated extensions and applications of three-way decisions [1, 7-10, 12, 13, 17-21, 23-29, $31,45,46,56,60-62,64-66]$.

The essential ideas of three-way decisions are commonly used in everyday life [32] and widely applied in many fields and disciplines, including, for example, medical decision-making [30,37,38], social judgement theory [39], hypothesis testing in statistics [42], management sciences [5,44], and peering review process [43]. However, a close examination surprisingly reveals that there still does not exist a unified formal description. To extend the concept of three-way decisions of rough sets to a much wider context, this paper outlines a theory of three-way decisions.

Information about this paper: Yao, Y.Y. An outline of a theory of threeway decisions. In: Yao, J., Yang, Y., Slowinski, R., Greco, S., Li, H., Mitra, S., Polkowski, L. (eds.) RSCTC 2012. LNCS (LNAI), vol. 7413, pp. 1-17. Springer, Heidelberg (2012) 


\section{A Description of Three-way Decisions}

The essential ideas of three-way decisions are described in terms of a ternary classification according to evaluations of a set of criteria.

Suppose $U$ is a finite nonempty set of objects or decision alternatives and $C$ is a finite set of conditions. Each condition in $C$ may be a criterion, an objective, or a constraint. For simplicity, in this paper we refer to conditions in $C$ as criteria. Our decision task is to classify objects of $U$ according to whether they satisfy the set of criteria. In widely used two-way decision models, it is assumed that an object either satisfies the criteria or does not satisfy the criteria. The set $U$ is divided into two disjoint regions, namely, the positive region POS for objects satisfying the criteria and the negative region NEG for objects not satisfying the criteria. There are usually some classification errors associated with such a binary classification. Two main difficulties with two-way approaches are their stringent binary assumption of the satisfiability of objects and the requirement of a dichotomous classification.

In many situations, it may happen that an object only satisfies the set of criteria to some degree. Even if an object may actually either satisfy or not satisfy the criteria, we may not be able to identify without uncertainty the subset of objects that satisfy the criteria due to uncertain or incomplete information. Consequently, we are only able to search for an approximate solution. Instead of making a binary decision, we use thresholds on the degrees of satisfiability to make one of three decisions: (a) accept an object as satisfying the set of criteria if its degree of satisfiability is at or above a certain level; (b) reject the object by treating it as not satisfying the criteria if its degree of satisfiability is at or below another level; and (c) neither accept nor reject the object but opt for a noncommitment. The third option may also be referred to as a deferment decision that requires further information or investigation. From the informal description, we give a formal definition.

The problem of three-way decisions. Suppose $U$ is a finite nonempty set and $C$ is a finite set of criteria. The problem of three-way decisions is to divide, based on the set of criteria $C, U$ into three pair-wise disjoint regions, POS, NEG, and BND, called the positive, negative, and boundary regions, respectively.

Corresponding to the three regions, one may construct rules for three-way decisions. In our previous studies $[52,54]$, we used three types of rules, namely, rules for acceptance, rejection, and noncommitment, respectively. It now appears to us that only rules for acceptance and rules for rejection are meaningful and sufficient. That is, the noncommitment set is formed by those objects to which neither a rule for acceptance nor a rule for rejection applies. It is not necessary to have, and in many cases may be impossible to construct, rules for noncommitement.

To formally describe the satisfiability of objects, rules for acceptance and rules for rejection, we need to introduce the notion of evaluations of objects and 
designated values for acceptance and designated values for rejection. Evaluations provide the degrees of satisfiability, designated values for acceptance are acceptable degrees of satisfiability, and designated valued for rejection are acceptable degrees of non-satisfiability. They provide a basis for a theory of three-way decisions.

A theory of three-way decisions must consider at least the following three issues regarding evaluations and designated values:

1. Construction and interpretation of a set of values for measuring satisfiability and a set of values for measuring non-satisfiability. The former is used by an evaluation for acceptance and the latter is used by an evaluation for rejection. In many cases, a single set may be used by both. It is assumed that the set of evaluation values is equipped with an ordering relation so that we can compare at least some objects according to their degrees of satisfiability or non-satisfiability. Examples of a set evaluation values are a poset, a lattice, a set of a finite numbers of grades, the set of integers, the unit interval, and the set of reals. Social judgement theory uses latitudes of acceptance, rejection, and noncommitment [6,39], which is closely related to our formulation of three-way decisions.

2. Construction and interpretation of evaluations. An evaluation depends on the set of criteria and characterizes either satisfiability or non-satisfiability of objects in $U$. Evaluations for the purposes of acceptance and rejection may be either independent or the same. Depending on particular applications, evaluations may be constructed and interpreted in terms of more intuitive and practically operable notions, including costs, risks, errors, profits, benefits, user satisfaction, committee voting, and so on. Based on the values of an evaluation, one can at least compare some objects.

3. Determination and interpretation of designated values for acceptance and designated values for rejection. The sets of designated values must meaningfully reflect an intuitive understanding of acceptance and rejection. For example, we can not accept and reject an object simultaneously. This requires that the set of designated values for acceptance and the set of designated value for rejection are disjoint. The designated values for acceptance should lead to monotonic decisions; if we accept an object $x$ then we should accept all those objects that have the same or larger degrees of satisfiability than $x$. It is also desirable if we can systematically determine the sets of designated values on a semantically sound basis.

By focusing on these issues, we examine three classes of evaluations. Evaluations are treated as a primitive notion for characterizing the satisfiability or desirability of objects. Their concrete physical interpretations are left to particular applications. 


\section{Evaluation-based Three-way Decisions}

We assume that evaluations for acceptance and rejection can be constructed based on the set of criteria. This enables us to focus mainly on how to obtain three-way decisions according to evaluations. The problem of constructing and interpreting evaluations is left to further studies and specific applications. A framework of evaluation-based three-way decisions is proposed and three models are introduced and studied.

\subsection{Three-way Decisions with a Pair of Poset-based Evaluations}

For the most general case, we consider a pair of (may be independent) evaluations, one for the purpose of acceptance and the other for rejection.

Definition 1. Suppose $U$ is a finite nonempty set and $\left(L_{a}, \preceq_{a}\right)\left(L_{r}, \preceq_{r}\right)$ are two posets. A pair of functions $v_{a}: U \longrightarrow L_{a}$ and $v_{r}: U \longrightarrow L_{r}$ is called an acceptance evaluation and a rejection evaluation, respectively. For $x \in U, v_{a}(x)$ and $v_{r}(x)$ are called the acceptance and rejection values of $x$, respectively.

In real applications, the set of possible values of acceptance may be interpreted based on more operational notions such as our confidence of an object satisfying the given set of criteria, or cost, benefit, and value induced by the object. For two objects $x, y \in U$, if $v_{a}(x) \preceq_{a} v_{a}(y)$, we say that $x$ is less acceptable than $y$. By adopting a poset $\left(L_{a}, \preceq_{a}\right)$, we assume that some objects in $U$ are incomparable. Similar interpretation can be said about the possible values of an evaluation for rejection. In general, acceptance and rejection evaluations may be independent.

To accept an object, its value $v_{a}(x)$ must be in a certain subset of $L_{a}$ representing the acceptance region of $L_{a}$. Similarly, we need to define the rejection region of $L_{r}$. By adopting a similar terminology of designated values in manyvalued logics [4], these values are called designated values for acceptance and designated values for rejection, respectively. Based on the two sets of designated values, one can easily obtain three regions for three-way decisions.

Definition 2. Let $\emptyset \neq L_{a}^{+} \subseteq L_{a}$ be a subset of $L_{a}$ called the designated values for acceptance, and $\emptyset \neq L_{r}^{-} \subseteq L_{r}$ be a subset of $L_{r}$ called the designated values for rejection. The positive, negative, and boundary regions of three-way decisions induced by $\left(v_{a}, v_{r}\right)$ are defined by:

$$
\begin{aligned}
& \operatorname{POS}_{\left(L_{a}^{+}, L_{r}^{-}\right)}\left(v_{a}, v_{r}\right)=\left\{x \in U \mid v_{a}(x) \in L_{a}^{+} \wedge v_{r}(x) \notin L_{r}^{-}\right\}, \\
& \operatorname{NEG}_{\left(L_{a}^{+}, L_{r}^{-}\right)}\left(v_{a}, v_{r}\right)=\left\{x \in U \mid v_{a}(x) \notin L_{a}^{+} \wedge v_{r}(x) \in L_{r}^{-}\right\}, \\
& \operatorname{BND}_{\left(L_{a}^{+}, L_{r}^{-}\right)}\left(v_{a}, v_{r}\right)=\left(\mathrm{POS}_{\left(L_{a}^{+}, L_{r}^{-}\right)}\left(v_{a}, v_{r}\right) \cup \mathrm{NEG}_{\left(L_{a}^{+}, L_{r}^{-}\right)}\left(v_{a}, v_{r}\right)\right)^{c} \\
&=\left\{x \in U \mid\left(v_{a}(x) \notin L_{a}^{+} \wedge v_{r}(x) \notin L_{r}^{-}\right) \vee\right. \\
&\left.\quad\left(v_{a}(x) \in L_{a}^{+} \wedge v_{r}(x) \in L_{r}^{-}\right)\right\} .
\end{aligned}
$$


The boundary region is defined as the complement of the union of positive and negative regions. The conditions in the definition of the positive and negative regions make sure that they are disjoint. Therefore, the three regions are pairwise disjoint. The three regions do not necessarily form a partition of $U$, as some of them may be empty. In fact, two-way decisions may be viewed as a special case of three-way decisions in which the boundary region is always empty.

By the interpretation of the orderings $\preceq_{a}$ and $\preceq_{r}$, the designated values $L_{a}^{+}$ for acceptance and the designated values $L_{r}^{-}$for rejection must satisfy certain properties. If $L_{a}$ has the largest element $\mathbf{1}$, then $\mathbf{1} \in L_{a}^{+}$. If $w \preceq_{a} u$ and $w \in L_{a}^{+}$, then $u \in L_{a}^{+}$. That is, if $v_{a}(x) \preceq_{a} v_{a}(y)$ and we accept $x$, then we must accept $y$. Similarly, if $L_{r}$ has the largest element $\mathbf{1}$, then $\mathbf{1} \in L_{r}^{-}$. If $w \preceq_{r} u$ and $w \in L_{r}^{-}$, then $u \in L_{r}^{-}$.

\subsection{Three-way Decisions with One Poset-based Evaluation}

In some situations, it may be more convenient to combine the two evaluation into a single acceptance-rejection evaluation. In this case, one poset $(L, \preceq)$ is used and two subsets of the poset are used as the designated values for acceptance and rejection, respectively.

Definition 3. Suppose $(L, \preceq)$ is a poset. A function $v: U \longrightarrow L$ is called an acceptance-rejection evaluation. Let $L^{+}, L^{-} \subseteq L$ be two subsets of $L$ with $L^{+} \cap L^{-}=\emptyset$, called the designated values for acceptance and the designated values for rejection, rspectively. The positive, negative, and boundary regions of three-way decisions induced by $v$ is defined by:

$$
\begin{aligned}
\operatorname{POS}_{\left(L^{+}, L^{-}\right)}(v) & =\left\{x \in U \mid v(x) \in L^{+}\right\}, \\
\operatorname{NEG}_{\left(L^{+}, L^{-}\right)}(v) & =\left\{x \in U \mid v(x) \in L^{-}\right\}, \\
\operatorname{BND}_{\left(L^{+}, L^{-}\right)}(v) & =\left\{x \in U \mid v(x) \notin L^{+} \wedge v(x) \notin L^{-}\right\} .
\end{aligned}
$$

The condition $L^{+} \cap L^{-}=\emptyset$ ensures that the three regions are pair-wise disjoint. A single evaluation $v$ may be viewed as a special case of two evaluations in which $\preceq_{a}=\preceq$ and $\preceq_{r}=\succeq$. In this way, acceptance is related to rejection in

the sense that the reverse ordering of acceptance is the ordering for rejection. To ensure the meaningfulness of $L^{+}$and $L^{-}$, it is required that $\neg(w \preceq u)$ for all $w \in L^{+}$and $u \in L^{-}$. In other words, $L^{+}$contains larger elements of $L$ and $L^{-}$ contains smaller elements of $L$.

\subsection{Three-way Decisions with an Evaluation Using a Totally Ordered Set}

Consider now an evaluation based on a totally ordered set $(L, \preceq)$ where $\preceq$ is a total order. That is, $\preceq$ is a partial order and any two elements of $L$ are comparable. This is in fact a widely used approach. For example, $L$ is either the set of real numbers or the unit interval $[0,1]$ and $\preceq$ is the less-than-or-equal relation $\leq$. For a total order, it is possible to define the sets of designated values for acceptance and rejection by a pair of thresholds. 
Definition 4. Suppose $(L, \preceq)$ is a totally ordered set, that is, $\preceq$ is a total order. For two elements $\alpha, \beta$ with $\beta \prec \alpha$ (i.e., $\beta \preceq \alpha \wedge \neg(\alpha \preceq \beta)$ ), suppose that the set of designated values for acceptance is given by $L^{+}=\{t \in L \mid t \succeq \alpha\}$ and the set of designated values for rejection is given by $L^{-}=\{b \in L \mid b \preceq \beta\}$. For an evaluation function $v: U \longrightarrow L$, its three regions are defined by:

$$
\begin{aligned}
\operatorname{POS}_{(\alpha, \beta)}(v) & =\{x \in U \mid v(x) \succeq \alpha\}, \\
\operatorname{NEG}_{(\alpha, \beta)}(v) & =\{x \in U \mid v(x) \preceq \beta\}, \\
\operatorname{BND}_{(\alpha, \beta)}(v) & =\{x \in U \mid \beta \prec v(x) \prec \alpha\} .
\end{aligned}
$$

Although evaluations based on a total order are restrictive, they have a computational advantage. One can obtain the three regions by simply comparing the evaluation value with a pair of thresholds. It is therefore not surprising to find that many studies in fact use a total order.

\subsection{Comments on Evaluations and Designated Values}

Construction and interpretation of evaluations and designated values are vital for practical applications of three-way decisions. At a theoretical level, it may be only possible to discuss required properties of evaluations. It is assumed that an evaluation is determined by a set of criteria, representing costs, benefits, degrees of desirability, objectives, constraints, and so on. Further studies on evaluations may be a fruitful research direction.

As an illustration, consider a simple linear model for constructing an evaluation. Suppose $C=\left\{c_{1}, c_{2}, \ldots, c_{m}\right\}$ are a set of $m$ criteria. Suppose $v_{c_{i}}: U \longrightarrow \Re$ denotes an evaluation based on criterion $v_{i}, 1 \leq i \leq m$. An overall evaluation function $v: U \longrightarrow \Re$ may be simply defined by a linear combination of individual evaluations:

$$
v(x)=v_{c_{1}}(x)+v_{c_{2}}(x)+\ldots+v_{c_{m}}(x) .
$$

Details of this linear utility model and other models can be found in literature of multi-crieria and multi-objective decision making [14].

Construction and interpretation of designated values may be explained in terms of benefits or risks of the resulting three regions of three-way decisions. For example, consider the model that uses a total order. Let $R_{P}(\alpha, \beta), R_{N}(\alpha, \beta)$ and $R_{B}(\alpha, \beta)$ denote the risks of the positive, negative, and boundary regions, respectively. It is reasonable to require that the sets of designated values are chosen to minimize the following overall risks:

$$
R(\alpha, \beta)=R_{P}(\alpha, \beta)+R_{N}(\alpha, \beta)+R_{B}(\alpha, \beta) .
$$

That is, finding a pair of thresholds can be formulated as the following optimization problem:

$$
\arg \min _{(\alpha, \beta)} R(\alpha, \beta) .
$$

As a concerte example, $R$ may be understood as uncertainty associated with three regions, by minimizing the overall uncertainty one can obtain the set of 
designed values in a probabilistic rough set model [2]. Two additional examples will be given in the next section when reviewing decision-theoretic rough sets [50, $57,58]$ and shadowed sets $[34,35]$.

\section{Models of Three-way Decisions}

We show that many studies on three-way decisions can be formulated within the framework proposed in the last section. For simplicity and as examples, we focus on the concept of concepts in a set-theoretical setting. In the classical view of concepts [40,41], every concept is understood as a unit of thought consisting of two parts, the intension and the extension of the concept. Due to uncertain or insufficient information, it is not always possible to precisely have a set of objects as the extension of a concept. Consequently, many generalizations of sets have been proposed and studied.

\subsection{Interval Sets and Three-valued Logic}

Interval sets provide a means to describe partially known concepts $[47,53]$. On the one hand, it is assumed that an object may actually be either an instance or not an instance of a concept. On the other hand, due to a lack of information and knowledge, one can only express the state of instance and non-instance for some objects, instead of all objects. That is, one has a partially known concept defined by a lower bound and upper bound of its extension.

Formally, a closed interval set is a subset of $2^{U}$ of the form,

$$
\left[A_{l}, A_{u}\right]=\left\{A \in 2^{U} \mid A_{l} \subseteq A \subseteq A_{u}\right\},
$$

where it is assumed that $A_{l} \subseteq A_{u}$, and $A_{l}$ and $A_{u}$ are called the lower and upper bound, respectively. Any set $X \in\left[A_{l}, A_{u}\right]$ may be the actual extension of the partially known concept. Constructive methods for defining interval sets can be formulated within an incomplete information table [16,22].

An interval set is an interval of the power set lattice $2^{U}$; it is also a lattice, with the minimum element $A_{l}$, the maximum element $A_{u}$, and the standard set-theoretic operations.

Interval-set algebra is related to Kleene's three-valued logic $[15,36]$, in which a third truth value is added to the standard two-valued logic. The third value may be interpreted as unknown or undeterminable. Let $L=\{F, I, T\}$ denote the set of truth values with a total order $F \preceq I \preceq T$. An interval set $\left[A_{l}, A_{u}\right]$ can be equivalently defined by an acceptance-rejection evaluation as,

$$
v_{\left[A_{l}, A_{u}\right]}(x)= \begin{cases}F, & x \in\left(A_{u}\right)^{c}, \\ I, & x \in A_{u}-A_{l}, \\ T, & x \in A_{l} .\end{cases}
$$

Suppose the sets of designated values for acceptance and rejection are defined by a pair of thresholds $(T, F)$, namely, $L^{+}=\{a \in L \mid T \preceq a\}=\{T\}$ and 
$L^{-}=\{b \in L \mid b \preceq F\}=\{F\}$. According to Definition 4, an interval set provides the following three-way decisions:

$$
\begin{aligned}
\operatorname{POS}_{(T, F)}\left(\left[A_{l}, A_{u}\right]\right) & =\left\{x \in U \mid v_{\left[A_{l}, A_{u}\right]}(x) \succeq T\right\}=A_{l}, \\
\operatorname{NEG}_{(T, F)}\left(\left[A_{l}, A_{u}\right]\right) & =\left\{x \in U \mid v_{\left[A_{l}, A_{u}\right]}(x) \preceq F\right\}=\left(A_{u}\right)^{c}, \\
\operatorname{BND}_{(T, F)}\left(\left[A_{l}, A_{u}\right]\right) & =\left\{x \in U \mid F \prec v_{\left[A_{l}, A_{u}\right]}(x) \prec T\right\}=A_{u}-A_{l} .
\end{aligned}
$$

Although the re-expression of an interval set in terms of three-way decisions is somewhat trivial, it does provide a new view to look at interval sets.

\subsection{Pawlak Rough Sets}

Pawlak rough set theory deals with approximations of a concept based on a family of definable concepts [33].

Let $E \subseteq U \times U$ denote an equivalence relation on $U$, that is, $E$ is reflexive, symmetric, and transitive. The equivalence class containing $x$ is defined by $[x]_{E}=[x]=\{y \in U \mid x E y\}$, which is a set of objects equivalent to $x$. The family of all equivalence classes of $E$ is called the quotient set induced by $E$, denoted as $U / E$. In an information table, an equivalence class is a definable set that can be defined by the conjunction of a family of attribute-value pairs [49].

For a subset $A \subseteq U$, the Pawlak rough set lower and upper approximations of $A$ are defined by:

$$
\begin{aligned}
\operatorname{apr}(A) & =\{x \in U \mid[x] \subseteq A\}, \\
\overline{\operatorname{apr}}(A) & =\{x \in U \mid[x] \cap A \neq \emptyset\} \\
& =\left\{x \in U \mid \neg\left([x] \subseteq A^{c}\right)\right\} .
\end{aligned}
$$

In the definition, we use an equivalent condition $\neg\left([x] \subseteq A^{c}\right)$ so that both lower and upper approximations are defined uniformly by using set inclusion $\subseteq$. According to the pair of approximations, the Pawlak positive, negative and boundary regions are defined by:

$$
\begin{aligned}
\operatorname{POS}(A) & =\underline{\operatorname{apr}}(A) \\
& =\{x \in U \mid[x] \subseteq A\} ; \\
\operatorname{NEG}(A) & =U-\overline{\operatorname{apr}}(A), \\
& =\left\{x \in U \mid[x] \subseteq A^{c}\right\} ; \\
\operatorname{BND}(A) & =\overline{\operatorname{apr}}(A)-\underline{\operatorname{apr}}(A), \\
& =\left\{x \in U \mid \neg\left([x] \subseteq A^{c}\right) \wedge \neg([x] \subseteq A)\right\} \\
& =(\operatorname{POS}(A) \cup \operatorname{NEG}(A))^{c} .
\end{aligned}
$$

Again, these regions are defined uniformly by using set inclusion. The three regions are pair-wise disjoint. Conversely, from the three regions, we can compute the pair of approximations by:

$$
\begin{aligned}
& \underline{\operatorname{apr}}(A)=\operatorname{POS}(A) \\
& \overline{\overline{a p r}}(A)=\operatorname{POS}(A) \cup \operatorname{BND}(A) .
\end{aligned}
$$


Therefore, rough set theory can be formulated by either a pair of approximations or three regions.

Three-way decisions with rough sets can be formulated as follows. Let $L_{a}=$ $L_{r}=\{F, T\}$ with $F \preceq T$, and let $L_{a}^{+}=L_{r}^{-}=\{T\}$. All objects in the same equivalence class have the same description. Based on descriptions of objects, we have a pair of an acceptance evaluation and a rejection evaluation:

$$
v_{(a, A)}(x)=\left\{\begin{array}{ll}
T, & {[x] \subseteq A,} \\
F, & \neg([x] \subseteq A) ;
\end{array} \quad v_{(r, A)}(x)= \begin{cases}T, & {[x] \subseteq A^{c},} \\
F, & \neg\left([x] \subseteq A^{c}\right) .\end{cases}\right.
$$

According to Definition 2, for a set $A \subseteq U$, we can make the following three-way decisions:

$$
\begin{aligned}
\operatorname{POS}_{(\{T\},\{T\})}(A) & =\left\{x \in U \mid v_{(a, A)}(x) \in\{T\} \wedge v_{(r, A)}(x) \notin\{T\}\right\} \\
& =\left\{x \in U \mid v_{(a, A)}(x)=T\right\} \\
& =\{x \in U \mid[x] \subseteq A\} \\
\operatorname{NEG}_{(\{T\},\{T\})}(A) & =\left\{x \in U \mid v_{(a, A)}(x) \notin\{T\} \wedge v_{(r, A)}(x) \in\{T\}\right\}, \\
& =\left\{x \in U \mid v_{(r, A)}(x)=T\right\} \\
& =\left\{x \in U \mid[x] \subseteq A^{c}\right\} \\
\operatorname{BND}_{(\{T\},\{T\})}(A) & =\left(\operatorname{POS}\left(v_{a}, v_{r}\right) \cup \operatorname{NEG}\left(v_{a}, v_{r}\right)\right)^{c} \\
& =\left\{x \in U \mid \neg([x] \subseteq A) \wedge \neg\left([x] \subseteq A^{c}\right)\right\} .
\end{aligned}
$$

The reformulation of rough set three regions based uniformly on set inclusion provides additional insights into rough set approximations. It explicitly shows that acceptance is based on an evaluation of the condition $[x] \subseteq A$ and rejection is based on an evaluation of the condition $[x] \subseteq A^{c}$. By those two conditions, both decisions of acceptance and rejection are made without any error. Whenever there is any doubt, namely, $\neg([x] \subseteq A) \wedge \neg\left([x] \subseteq A^{c}\right)$, a decision of noncommitment is made.

\subsection{Decision-Theoretic Rough Sets}

Decision-theoretic rough sets (DTRS) $[48,50,51,57,58]$ are a quantitative generalization of Pawlak rough sets by considering the degree of inclusion of an equivalence class in a set.

The acceptance-rejection evaluation used by a DTRS model is the conditional probability $v_{A}(x)=\operatorname{Pr}(A \mid[x])$, with values from the totally ordered set $([0,1], \leq)$. Given a pair of thresholds $(\alpha, \beta)$ with $0 \leq \beta<\alpha \leq 1$, the sets of designated values for acceptance and rejections are $L^{+}=\{a \in[0,1] \mid \alpha \leq a\}$

and $L^{-}=\{b \in[0,1] \mid b \leq \beta\}$. According to Definition 4, a DTRS model makes the following three-way decisions: for $A \subseteq U$,

$$
\begin{aligned}
\operatorname{POS}_{(\alpha, \beta)}(A) & =\left\{x \in U \mid v_{A}(x) \succeq \alpha\right\} \\
& =\{x \in U \mid \operatorname{Pr}(A \mid[x]) \geq \alpha\},
\end{aligned}
$$




$$
\begin{aligned}
\operatorname{NEG}_{(\alpha, \beta)}(A) & =\left\{x \in U \mid v_{A}(x) \preceq \beta\right\} \\
& =\{x \in U \mid \operatorname{Pr}(A \mid[x]) \leq \beta\}, \\
\operatorname{BND}_{(\alpha, \beta)}(A) & =\left\{x \in U \mid \beta \prec v_{A}(x) \prec \alpha\right\} \\
& =\{x \in U \mid \beta<\operatorname{Pr}(A \mid[x])<\alpha\} .
\end{aligned}
$$

Three-way decision-making in DTRS can be easily related to incorrect acceptance error and incorrect rejection error [55]. Specifically, incorrect acceptance error is given by $\operatorname{Pr}\left(A^{c} \mid[x]\right)=1-\operatorname{Pr}(A \mid[x]) \leq 1-\alpha$, which is bounded by $1-\alpha$. Likewise, incorrect rejection error is given by $\operatorname{Pr}(A \mid[x]) \leq \beta$, which is bounded by $\beta$. Therefore, the pair of thresholds can be interpreted as defining tolerance levels of errors.

A main advantage of a DTRS model is its solid foundation based on Bayesian decision theory. In addition, the pair of thresholds can be systematically computed by minimizing overall ternary classification cost [55].

Bayesian decision theory [3] can be applied to the derivation of DTRS as follows. We have a set of 2 states and a set of 3 actions for each state. The set of states is given by $\Omega=\left\{A, A^{c}\right\}$ indicating that an object is in $A$ and not in $A$, respectively. For simplicity, we use the same symbol to denote both a subset $A$ and the corresponding state. With respect to the three regions, the set of actions with respect to a state is given by $\mathcal{A}=\left\{a_{P}, a_{N}, a_{B}\right\}$, where $a_{P}, a_{N}$, and $a_{B}$ represent the three actions in classifying an object $x$, namely, deciding $x \in \operatorname{POS}(A)$, deciding $x \in \operatorname{NEG}(A)$, and deciding $x \in \operatorname{BND}(A)$, respectively. The losses regarding the risk or cost of those classification actions with respect to different states are given by the $3 \times 2$ matrix:

\begin{tabular}{|c|c|c|}
\hline & $A(P)$ & $A^{c}(N)$ \\
\hline$a_{P}$ & $\lambda_{P P}$ & $\lambda_{P N}$ \\
$a_{N}$ & $\lambda_{N P}$ & $\lambda_{N N}$ \\
$a_{B}$ & $\lambda_{B P}$ & $\lambda_{B N}$ \\
\hline
\end{tabular}

In the matrix, $\lambda_{P P}, \lambda_{N P}$ and $\lambda_{B P}$ denote the losses incurred for taking actions $a_{P}, a_{N}$ and $a_{B}$, respectively, when an object belongs to $A$, and $\lambda_{P N}, \lambda_{N N}$ and $\lambda_{B N}$ denote the losses incurred for taking the same actions when the object does not belong to $A$

To determine a pair of thresholds for three-way decisions, one can minimize the following overall risk $[12,55]$ :

$$
R(\alpha, \beta)=R_{P}(\alpha, \beta)+R_{N}(\alpha, \beta)+R_{B}(\alpha, \beta),
$$

where

$$
\begin{aligned}
& R_{P}(\alpha, \beta)=\sum_{\operatorname{Pr}(A \mid[x]) \geq \alpha}\left[\lambda_{P P} \operatorname{Pr}(A \mid[x])+\lambda_{P N} \operatorname{Pr}\left(A^{c} \mid[x]\right)\right] \operatorname{Pr}([x]), \\
& R_{N}(\alpha, \beta)=\sum_{\operatorname{Pr}(A \mid[x]) \leq \beta}\left[\lambda_{N P} \operatorname{Pr}(A \mid[x])+\lambda_{N N} \operatorname{Pr}\left(A^{c} \mid[x]\right)\right] \operatorname{Pr}([x]), \\
& R_{B}(\alpha, \beta)=\sum_{\beta<\operatorname{Pr}(A \mid[x])<\alpha}\left[\lambda_{B P} \operatorname{Pr}(A \mid[x])+\lambda_{B N} \operatorname{Pr}\left(A^{c} \mid[x]\right)\right] \operatorname{Pr}([x]),
\end{aligned}
$$


represent, risks incurred by acceptance, rejection, and noncommitment, and the summation is over all equivalence classes. It can be shown $[50,55]$ that under the following conditions,

$$
\begin{array}{ll}
\left(\mathrm{c}_{1}\right) \quad \lambda_{P P}<\lambda_{B P}<\lambda_{N P}, \quad \lambda_{N N}<\lambda_{B N}<\lambda_{P N}, \\
\left(\mathrm{c}_{2}\right) \quad\left(\lambda_{P N}-\lambda_{B N}\right)\left(\lambda_{N P}-\lambda_{B P}\right)>\left(\lambda_{B N}-\lambda_{N N}\right)\left(\lambda_{B P}-\lambda_{P P}\right),
\end{array}
$$

a pair of threshold $(\alpha, \beta)$ with $0 \leq \beta<\alpha \leq 1$ that minimizes $R$ is given by:

$$
\begin{aligned}
& \alpha=\frac{\left(\lambda_{P N}-\lambda_{B N}\right)}{\left(\lambda_{P N}-\lambda_{B N}\right)+\left(\lambda_{B P}-\lambda_{P P}\right)}, \\
& \beta=\frac{\left(\lambda_{B N}-\lambda_{N N}\right)}{\left(\lambda_{B N}-\lambda_{N N}\right)+\left(\lambda_{N P}-\lambda_{B P}\right)} .
\end{aligned}
$$

That is, the pair of thresholds can be computed from the loss function.

Other models for determining the pair of thresholds include a game-theoretic framework $[1,9,11]$, a multi-view decision model $[17,66]$, and the minimization of uncertainty of the three regions [2]. The conditional probability required by DTRS can be estimated based on a naive Bayesian rough set model [59] or a regression model [25].

\subsection{Three-valued Approximations in Many-valued Logic and Fuzzy Sets}

Three-valued approximations in many-valued logics are formulated based on the discussion given by Gottwald [4] on positively designated truth degrees and negatively designated truth degrees.

In many-valued logic, the set of truth degrees or values is normally an ordered set $(L, \preceq)$ and contains the classical truth values $F$ and $T$ (often coded by 0 and $1)$ as its minimum and maximum elements, namely, $\{F, T\} \subseteq L$ and for any $u \in L, F \preceq u \preceq T$. It is also a common practice to use a subset $L^{+}$of positively designated truth degrees to code the intuitive notion of truth and to use another subset $L^{-}$of negatively designated truth degrees to code the opposite. For the two sets to be meaningful, the following conditions are normally assumed [4]:

$$
\begin{array}{ll}
\text { (i) } \quad & T \in L^{+}, \\
& F \in L^{-}, \\
\text {(ii) } & L^{+} \cup L^{-} \subseteq L, \\
& L^{+} \cap L^{-}=\emptyset, \\
\text { (iii) } & w \preceq u \wedge w \in L^{+} \Longrightarrow u \in L^{+}, \\
& w \preceq u \wedge u \in L^{-} \Longrightarrow w \in L^{-} .
\end{array}
$$

Three-valued approximations of a many-valued logic derive from three-way decisions based on the two designated sets. We accept a truth degree as being true if it is in the positively designated set, reject it as being true if it is the negatively 
designated set, and neither accept nor reject if it is not in any of the two sets. By so doing, we can have a new three-valued logic with the set of truth values $L_{3}=\left\{L^{-}, L-\left(L^{-} \cup L^{+}\right), L^{+}\right\}$under the ordering $L^{-} \preceq_{3} L-\left(L^{-} \cup L^{+}\right) \preceq_{3} L^{+}$, which is an approximation of the many-valued logic.

To a large extent, our formulation of three-way decisions, as given in the last section, draws mainly from such a consideration. Specifically, we borrowed the notions of designated truth degrees from studies of many-valued logic to introduce the notions of designated values for acceptance and rejection in the theory of three-way decisions.

A fuzzy set $\mathcal{A}$ is characterized by a mapping from $U$ to the unit interval, namely, $\mu_{\mathcal{A}}: U \longrightarrow[0,1]$. The value $\mu_{\mathcal{A}}(x)$ is called the degree of membership of the object $x \in U$. Fuzzy sets may be interpreted in terms of a many-valued logic with the unit interval as its set of truth degrees. According to the three-valued approximations of a many-valued logic, one can similarly formulate three-valued approximations of a fuzzy set. This formulation was in fact given by Zadeh [63] in his seminal paper on fuzzy sets and was shown to be related to Kleene's three-valued logic.

Given a pair of thresholds $(\alpha, \beta)$ with $0 \leq \beta<\alpha \leq 1$, one can define the designated sets of values for acceptance and rejection as $L^{+}=\{a \in[0,1] \mid \alpha \leq a\}$ and $L^{-}=\{b \in[0,1] \mid b \leq \beta\}$. According to Definition 4 , if a fuzzy membership function $\mu_{\mathcal{A}}$ is used as an acceptance-rejection evaluation, namely, $v_{\mu_{\mathcal{A}}}=\mu_{\mathcal{A}}$, we have the following three-way decisions,

$$
\begin{aligned}
\operatorname{POS}_{(\alpha, \beta)}\left(\mu_{\mathcal{A}}\right) & =\left\{x \in U \mid v_{\mu_{\mathcal{A}}}(x) \succeq \alpha\right\} \\
& =\left\{x \in U \mid \mu_{\mathcal{A}}(x) \geq \alpha\right\}, \\
\operatorname{NEG}_{(\alpha, \beta)}\left(\mu_{\mathcal{A}}\right) & =\left\{x \in U \mid v_{\mu_{\mathcal{A}}}(x) \preceq \beta\right\} \\
& =\left\{x \in U \mid \mu_{\mathcal{A}}(x) \leq \beta\right\}, \\
\operatorname{BND}_{(\alpha, \beta)}\left(\mu_{\mathcal{A}}\right) & =\left\{x \in U \mid \beta \prec v_{\mu_{\mathcal{A}}}(x) \prec \alpha\right\} \\
& =\left\{x \in U \mid \beta<\mu_{\mathcal{A}}(x)<\alpha\right\} .
\end{aligned}
$$

Zadeh [63] provided an interpretation of this three-valued approximations of a fuzzy set: one may say that (1) $x$ belongs to $\mathcal{A}$ if $\mu_{\mathcal{A}}(x) \geq \alpha ;(2) x$ does not belong to $\mathcal{A}$ if $\mu_{\mathcal{A}}(x) \leq \beta$; and (3) $x$ has an indeterminate status relative to $\mathcal{A}$ if $\beta<\mu_{\mathcal{A}}(x)<\alpha$. This interpretation explicitly uses the notions of acceptance and rejection and is consistent with our three-way decisions.

\subsection{Shadowed Sets}

In contrast to decision-theoretic rough sets in which the pair of thresholds can be interpreted by classification errors, there is a difficulty in interpreting thresholds in three-valued approximations of a fuzzy sets. The introduction of a shadowed set induced by a fuzzy set attempts to address this problem $[34,35]$.

A shadowed set $\mathbb{A}$ is defined as a mapping, $S_{\mathbb{A}}: U \longrightarrow\{0,[0,1], 1\}$, from $U$ to a set of three truth values. It is assumed that the three values are ordered by $0 \preceq[0,1] \preceq 1$. The value $[0,1]$ represents the membership of objects in the 
shadows of a shadowed set. Like the interval-set algebra, shadowed-set algebra is also related to Kleene's three-valed logic. Shadowed sets provide another model of three-way decisions.

Unlike an interval set, a shadowed set is constructed from a fuzzy set $\mu_{\mathcal{A}}$ : $U \longrightarrow[0,1]$ as follows:

$$
S_{\mathbb{A}}(x)= \begin{cases}0, & \mu_{\mathcal{A}}(x) \leq \tau, \\ {[0,1],} & \tau<\mu_{\mathcal{A}}(x)<1-\tau, \\ 1, & \mu_{\mathcal{A}}(x) \geq 1-\tau,\end{cases}
$$

where $0 \leq \tau<0.5$ is a threshold. Given a pair of thresholds $(1,0)$ for the set of truth values $\{0,[0,1], 1\}$, by Definition 4 and equations (19) and (20), we have the following three-way decision for a shadowed set:

$$
\begin{aligned}
\operatorname{POS}_{(1,0)}\left(S_{\mathbb{A}}\right) & =\left\{x \in U \mid v_{S_{\mathbb{A}}}(x) \succeq 1\right\} \\
& =\left\{x \in U \mid \mu_{\mathcal{A}}(x) \geq 1-\tau\right\} \\
& =\operatorname{POS}_{(1-\tau, \tau)}\left(\mu_{\mathcal{A}}\right), \\
\operatorname{NEG}_{(1,0)}\left(S_{\mathbb{A}}\right) & =\left\{x \in U \mid v_{S_{\mathbb{A}}}(x) \preceq 0\right\} \\
& =\left\{x \in U \mid \mu_{\mathcal{A}}(x) \leq \tau\right\}, \\
& =\operatorname{NEG}_{(1-\tau, \tau)}\left(\mu_{\mathcal{A}}\right), \\
\operatorname{BND}_{(1,0)}\left(S_{\mathbb{A}}\right) & =\left\{x \in U \mid 0 \prec v_{S_{\mathbb{A}}}(x) \prec 1\right\} \\
& =\left\{x \in U \mid \tau<\mu_{\mathcal{A}}(x)<1-\tau\right\} \\
& =\operatorname{BND}_{(1-\tau, \tau)}\left(\mu_{\mathcal{A}}\right) .
\end{aligned}
$$

That is, a shadowed set is a three-valued approximation of a fuzzy set with $(\alpha, \beta)=(1-\tau, \tau)$. In general, one can also consider shadowed set by a pair of thresholds $(\alpha, \beta)$ with $0 \leq \beta<\alpha \leq 1$ on a fuzzy set $\mu_{\mathcal{A}}$.

As shown in $[34,35]$, the threshold $\tau$ for constructing a shadowed set can be determined by minimizing the following function,

$$
\Omega(\tau)=\operatorname{abs}\left(\Omega_{r}(\tau)+\Omega_{e}(\tau)-\Omega_{s}(\tau)\right),
$$

where $\operatorname{abs}(\cdot)$ stands for the absolute value and

$$
\begin{aligned}
& \Omega_{r}(\tau)=\sum_{\left\{x \in U \mid \mu_{\mathcal{A}}(x) \leq \tau\right\}} \mu_{\mathcal{A}}(x), \\
& \Omega_{e}(\tau)=\sum_{\left\{y \in U \mid \mu_{\mathcal{A}}(y) \geq 1-\tau\right\}}\left(1-\mu_{\mathcal{A}}(y)\right), \\
& \Omega_{s}(\tau)=\operatorname{card}\left(\left\{z \in U \mid \tau<\mu_{\mathcal{A}}(z)<1-\tau\right\}\right),
\end{aligned}
$$

are, respectively, the total of reduced membership values from $\mu_{\mathcal{A}}(x)$ in the fuzzy set to 0 in the shadowed set (i.e., $\mu_{\mathcal{A}}(x)-0=\mu_{\mathcal{A}}(x)$ ), the total of elevated membership values from $\mu_{\mathcal{A}}(y)$ in the fuzzy set to 1 in the shadowed set (i.e., $1-$ $\mu_{\mathcal{A}}(y)$ ), and the cardinality of the shadows of the shadowed set. The minimization of $\Omega(\tau)$ may be equivalently formulated as finding a solution to the equation,

$$
\Omega_{r}(\tau)+\Omega_{e}(\tau)=\Omega_{s}(\tau)
$$


if it has a solution. Although the problem of finding the threshold $\tau$ is formulated precisely, the meaning of the objective function $\Omega(\tau)$ still needs further investigation. It is interesting to note that the objective function $\Omega(\tau)$ shares some similarity to the objective function $R(\alpha, \beta)$ of a DTRS model, which may shed some light on the problem of determining the threshold in shadowed sets.

\section{Conclusions}

The concept of three-way decisions provides an appealing interpretation of three regions in probabilistic rough sets. The positive and negative regions are sets of accepted objects and rejected objects, respectively. The boundary region is the set of objects for which neither acceptance nor rejection is possible, due to uncertain or incomplete information. A close examination of studies and applications of three-way decisions shows that (a) essential ideas of three-way decisions are general applicable to a wide range of decision-making problems; (b) we routinely make three-way decisions in everyday life; (c) three-way decisions appear across many fields and disciplines; and (d) there is a lack of formal theory for three-way decisions. These findings motivate a study of a theory of three-way decisions in its own right.

We outline a theory of three-way decisions based on the notions of evaluations for acceptance and evaluations for rejection. One accepts or rejects an object when its values from evaluations fall into some designated areas; otherwise, one makes a decision of noncommitment. We propose and study three classes of evaluations. We demonstrate that the proposed theory can describe and explain three-way decisions in many-valued logics and generalizations of set theory, including interval sets, rough sets, decision-theoretic rough sets, fuzzy sets, and shadowed sets. As future research, we plan to investigate three-way decisions in other settings.

\section{Acknowledgements}

This work is partially supported by a Discovery Grant from NSERC Canada.

\section{References}

1. Azam, N., Yao, J.T.: Multiple criteria decision analysis with game-theoretic rough sets. In: Li, T., Nguyen, H.S., Wang, G., Grzymala-Busse, J.W., Janicki, R., Hassanien, A.E., Yu, H. (eds.) RSKT 2012. LNCS (LNAI), vol. 7414, pp. 399-408. Springer, Heidelberg (2012)

2. Deng, X.F., Yao, Y.Y.: An information-theoretic interpretation of thresholds in probabilistic rough sets. In: Li, T., Nguyen, H.S., Wang, G., Grzymala-Busse, J.W., Janicki, R., Hassanien, A.E., Yu, H. (eds.) RSKT 2012. LNCS (LNAI), vol. 7414, pp. 369-378. Springer, Heidelberg (2012)

3. Duda, R.O., Hart, P.E.: Pattern Classification and Scene Analysis. Wiley, New York (1973) 
4. Gottwald, S.: A Treatise on Many-Valued Logics. Research Studies Press, Baldock, Hertfordshire, England (2001)

5. Goudey, R.: Do statistical inferences allowing three alternative decision give better feedback for environmentally precautionary decision-making. Journal of Environmental Management 85, 338-344 (2007)

6. Granberg, D., Steele, L.: Procedural considerations in measuring latitudes of acceptance, rejection, and noncommitment. Social Forces 52, 538-542 (1974)

7. Grzymala-Busse, J.W.: Generalized parameterized approximations. In: Yao, J., Ramanna, S., Wang, G., Suraj, Z. (eds.) RSKT 2011. LNCS (LNAI), vol. 6954, pp. 136-145. Springer, Heidelberg (2011)

8. Grzymala-Busse, J.W., Yao, Y.Y.: Probabilistic rule induction with the LERS data mining system. International Journal of Intelligent Systems 26, 518-539 (2011)

9. Herbert, J.P., Yao, J.T.: Learning optimal parameters in decision-theoretic rough sets. In: Wen, P., Li, Y., Polkowski, L., Yao, Y., Tsumoto, H., Wang, G. (eds.) RSKT 2009. LNCS (LNAI), vol. 5589, pp. 610-617. Springer, Heidelberg (2009)

10. Herbert, J.P., Yao, J.T.: Game-theoretic rough sets. Fundamenta Informaticae 108, 267-286 (2011)

11. Herbert, J.P., Yao, J.T.: Analysis of data-driven parameters in game-theoretic rough sets. In: Yao, J., Ramanna, S., Wang, G., Suraj, Z. (eds.) RSKT 2011. LNCS (LNAI), vol. 6954, pp. 447-456. Springer, Heidelberg (2011)

12. Jia, X.Y., Li, W.W., Shang, L., Chen, J.J.: An optimization viewpoint of decisiontheoretic rough set model. In: Yao, J., Ramanna, S., Wang, G., Suraj, Z. (eds.) RSKT 2011. LNCS (LNAI), vol. 6954, pp. 457-465. Springer, Heidelberg (2011)

13. Jia, X.Y., Zhang, K., Shang, L.: Three-way decisions solution to filter spam email: An empirical study. In: Yao, J., Yang, Y., Slowinski, R., Greco, S., Li, H., Mitra, S., Polkowski, L. (eds.) RSCTC 2012. LNCS (LNAI), vol. 7413, pp. 287-296. Springer, Heidelberg (2012)

14. Keeney, R.L., Raiffa, H.: Decisions With Multiple Objectives: Preferences and Value Tradeoffs. John Wiley and Sons, New York (1976)

15. Kleene, S.C.: Introduction to Mathematics. Groningen, New York (1952)

16. Li, H.X., Wang, M.H., Zhou, X.Z., Zhao, J.B.: An interval set model for learning rules from incomplete information table. International Journal of Approximate Reasoning 53, 24-37 (2012)

17. Li, H.X., Zhou, X.Z.: Risk decision making based on decision-theoretic rough set: A three-way view decision model. International Journal of Computational Intelligence Systems 4, 1-11 (2011)

18. Li, H.X., Zhou, X.Z., Li, T.R., Wang, G.Y., Miao, D.Q., Yao, Y.Y. (eds.): DecisionTheoretic Rough Sets Theory and Recent Research (in Chinese). Science Press, Beijing (2011)

19. Li, H.X., Zhou, X.Z., Zhao, J.B., Huang, B.: Cost-sensitive classification based on decision-theoretic rough set model. In: Li, T., Nguyen, H.S., Wang, G., GrzymalaBusse, J.W., Janicki, R., Hassanien, A.E., Yu, H. (eds.) RSKT 2012. LNCS (LNAI), vol. 7414, pp. 379-388. Springer, Heidelberg (2012)

20. Li, H.X., Zhou, X.Z., Zhao, J.B., Liu, D.: Attribute reduction in decision-theoretic rough set model: A further investigation. In: Yao, J., Ramanna, S., Wang, G., Suraj, Z. (eds.) RSKT 2011. LNCS (LNAI), vol. 6954, pp. 466-475. Springer, Heidelberg (2011)

21. Li, W., Miao, D.Q., Wang, W.L., Zhang, N.: Hierarchical rough decision theoretic framework for text classification. In: Proceedings of the 9th IEEE International Conference on Cognitive Informatics, pp. 484-489 (2009) 
22. Lipski, W. Jr.: On semantic issues connected with incomplete information databases. ACM Transactions on Database Systems 4, 269-296 (1979)

23. Liu, D., Li, H.X., Zhou, X.Z.: Two decades' research on decision-theoretic rough sets. In: Proceedings of the 9th IEEE International Conference on Cognitive Informatics, pp. 968-973 (2010).

24. Liu, D., Li, T.R., Li, H.X.: A multiple-category classification approach with decision-theoretic rough sets. Fundamenta Informaticae 115, 173-188 (2012)

25. Liu, D., Li, T.R., Liang, D.C.: A new discriminant analysis approach under decision-theoretic rough sets. In: Yao, J., Ramanna, S., Wang, G., Suraj, Z. (eds.) RSKT 2011. LNCS (LNAI), vol. 6954, pp. 476-485. Springer, Heidelberg (2011)

26. Liu, D., Li, T.R., Liang, D.C.: Decision-theoretic rough sets with probabilistic distribution. In: Li, T., Nguyen, H.S., Wang, G., Grzymala-Busse, J.W., Janicki, R., Hassanien, A.E., Yu, H. (eds.) RSKT 2012. LNCS (LNAI), vol. 7414, pp. 389398. Springer, Heidelberg (2012)

27. Liu, D., Li, T.R., Ruan, D.: Probabilistic model criteria with decision-theoretic rough sets. Information Sciences 181, 3709-3722 (2011)

28. Liu, D., Yao, Y.Y., Li, T.R.: Three-way investment decisions with decisiontheoretic rough sets. International Journal of Computational Intelligence Systems 4, 66-74 (2011)

29. Liu, J.B., Min, F., Liao, S.J., Zhu, W.: Minimal test cost feature selection with positive region constraint. In: Yao, J., Yang, Y., Slowinski, R., Greco, S., Li, H., Mitra, S., Polkowski, L. (eds.) RSCTC 2012. LNCS (LNAI), vol. 7413, pp. 259-266. Springer, Heidelberg (2012)

30. Lurie, J.D., Sox, H.C.: Principles of medical decision making. Spine 24, 493-498 (1999)

31. Ma, X.A., Wang, G.Y., Yu, H.: Multiple-category attribute reduct using decisiontheoretic rough set model. In: Yao, J., Yang, Y., Slowinski, R., Greco, S., Li, H., Mitra, S., Polkowski, L. (eds.) RSCTC 2012. LNCS (LNAI), vol. 7413, pp. 267-276. Springer, Heidelberg (2012)

32. Marinoff, L.: The Middle Way, Finding Happiness in a World of Extremes. Sterling, New York (2007)

33. Pawlak, Z. Rough sets, International Journal of Computer and Information Sciences 11, 341-356 (1982)

34. Pedrycz, W.: Shadowed sets: Representing and processing fuzzy sets. IEEE Transactions on Systems, Man, and Cybernetics, Part B: Cybernetics 28, 103-109 (1998)

35. Pedrycz, W.: ¿From fuzzy sets to shadowed sets: Interpretation and computing. International Journal of Intelligent Systems 24, 48-61 (2009)

36. Rescher, N.: Many-valued Logic. McGraw-Hill, New York (1969)

37. Pauker, S.G., Kassirer, J.P.: The threshold approach to clinical decision making. The New England Journal of Medicine 302, 1109-1117 (1980)

38. Schechter, C.B.: Sequential analysis in a Bayesian model of diastolic blood pressure measurement. Medical Decision Making 8, 191-196 (1988)

39. Sherif, M., Hovland, C.I.: Social Judgment: Assimilation and Contrast Effects in Communication and Attitude Change. Yale University Press, New Haven (1961)

40. Smith, E.E.: Concepts and induction. In: Posner, M.I. (ed.) Foundations of Cognitive Science, pp. 501-526. The MIT Press, Cambridge, Massachusetts (1989)

41. van Mechelen, I., Hampton, J., Michalski, R.S., Theuns, P. (eds.): Categories and Concepts, Theoretical Views and Inductive Data Analysis. Academic Press, New York (1993)

42. Wald, A.: Sequential tests of statistical hypotheses. The Annals of Mathematical Statistics 16, 117-186 (1945) 
43. Weller, A.C.: Editorial Peer Review: Its Strengths and Weaknesses. Information Today, Inc., Medford, NJ (2001)

44. Woodward, P.W., Naylor, J.C.: An application of Bayesian methods in SPC. The Statistician 42, 461-469 (1993)

45. Yang, X.P., Song, H.G., Li, T.J.: Decision making in incomplete information system based on decision-theoretic rough sets. In: Yao, J., Ramanna, S., Wang, G., Suraj, Z. (eds.) RSKT 2011. LNCS (LNAI), vol. 6954, pp. 495-503. Springer, Heidelberg (2011)

46. Yang, X.P., Yao, J.T: Modelling multi-agent three-way decisions with decisiontheoretic rough sets. Fundamenta Informaticae 115, 157-171 (2012)

47. Yao, Y.Y.: Interval-set algebra for qualitative knowledge representation. In: Proceedings of the 5th International Conference on Computing and Information, pp. 370-374 (1993)

48. Yao, Y.Y.: Probabilistic approaches to rough sets. Expert Systems 20, 287-297 (2003)

49. Yao, Y.Y.: A note on definability and approximations. LNCS Transactions on Rough Sets VII, LNCS, vol. 4400, 274-282 (2007)

50. Yao, Y.Y.: Decision-theoretic rough set models. In: Yao, J., Lingras, P., Wu, W.Z., Szczuka, M.S., Cercone, N., Slezak, D. (eds.) RSKT 2007. LNCS (LNAI), vol. 4481, pp. 1-12, Springer, Heidelberg (2007)

51. Yao, Y.Y.: Probabilistic rough set approximations. International Journal of Approximation Reasoning 49, 255-271 (2008)

52. Yao, Y.Y.: Three-way decision: An interpretation of rules in rough set theory. In: Wen, P., Li, Y., Polkowski, L., Yao, Y., Tsumoto, H., Wang, G. (eds.) RSKT 2009. LNCS (LNAI), vol. 5589, pp. 642-649. Springer, Heidelberg (2009)

53. Yao, Y.Y.: Interval sets and interval-set algebras. In: Proceedings of the 8th IEEE International Conference on Cognitive Informatics, pp. 307-314 (2009)

54. Yao, Y.Y.: Three-way decisions with probabilistic rough sets. Information Sciences 180, 341-353 (2010)

55. Yao, Y.Y.: The superiority of three-way decisions in probabilistic rough set models. Information Sciences 181, 1080-1096 (2011)

56. Yao, Y.Y., Deng, X.F.: Sequential three-way decisions with probabilistic rough sets. In: Proceedings of the 10th IEEE International Conference on Cognitive Informatics \& Cognitive Computing, pp. 120-125 (2011)

57. Yao, Y.Y., Wong, S.K.M.: A decision theoretic framework for approximating concepts. International Journal of Man-machine Studies 37, 793-809 (1992)

58. Yao, Y.Y., Wong, S.K.M., Lingras, P.: A decision-theoretic rough set model. In: Z.W. Ras, M. Zemankova and M.L. Emrich (eds.) Methodologies for Intelligent Systems 5, pp. 17-24. North-Holland, New York (1990)

59. Yao, Y.Y., Zhou, B.: Naive Bayesian rough sets. In: Yu, J., Greco, S., Lingras, P., Wang, G., Skowron, A. (eds.) RSKT 2010. LNCS (LNAI), vol. 6401, pp. 719-726. Springer, Heidelberg (2010)

60. Yu, H., Chu, S.S., Yang, D.C.: Autonomous knowledge-oriented clustering using decision-theoretic rough set theory. Fundamenta Informaticae 115, 141-156 (2012)

61. Yu, H., Liu, Z.G., Wang, G.Y.: Automatically determining the number of clusters using decision-theoretic rough set. In: Yao, J., Ramanna, S., Wang, G., Suraj, Z. (eds.) RSKT 2011. LNCS (LNAI), vol. 6954, pp. 504-513. Springer, Heidelberg (2011)

62. Yu, H., Wang, Y.: Three-way decisions method for overlapping clustering. In: Yao, J., Yang, Y., Slowinski, R., Greco, S., Li, H., Mitra, S., Polkowski, L. (eds.) RSCTC 2012. LNCS (LNAI), vol. 7413, pp. 277-286. Springer, Heidelberg (2012) 
63. Zadeh, L.A.: Fuzzy sets. Information and Control 8, 338-353 (1965)

64. Zhou, B.: A new formulation of multi-category decision-theoretic rough sets. In: Yao, J., Ramanna, S., Wang, G., Suraj, Z. (eds.) RSKT 2011. LNCS (LNAI), vol. 6954, pp. 514-522. Springer, Heidelberg (2011)

65. Zhou, B., Yao, Y.Y., Luo, J.G.: A three-way decision approach to email spam filtering. In: Farzindar, A., Keselj, V. (eds.) AI 2010. LNCS (LNAI), vol. 6085, pp. 28-39. Springer, Heidelberg (2010)

66. Zhou, X.Z., Li, H.X.: A multi-view decision model based on decision-theoretic rough set. In: Wen, P., Li, Y., Polkowski, L., Yao, Y., Tsumoto, H., Wang, G. (eds.) RSKT 2009. LNCS (LNAI), vol. 5589, pp. 650-657. Springer, Heidelberg (2009) 